\title{
Why is Pharma So Scared of Psychiatric Drug Discovery?
}

\author{
Mark D Tricklebank ${ }^{1 *}$, Laura A Ajram² \\ ${ }^{1}$ Department of Neuroimaging, Institute of Psychiatry, Psychology and Neuroscience, Kings College London, London, UK \\ ${ }^{2}$ Department of Forensic and Neurodevelopmental Sciences, The Sackler Centre for Translational Neurodevelopment, \\ Institute of Psychiatry, Psychology \& Neuroscience, King's College London, London, UK
}

Keywords: Drug discovery, Pharmaceutical industry, Molecular drug targets, Opioids

Accepted on May 03, 2017

\section{Introduction}

The 1950s heralded a golden age for psychiatric drug discovery; for the first time, compounds capable of treating serious mental disturbances were found that, despite their shortcomings, proved the pharmacotherapy of mental disorders was both possible and effective. Not only did these early treatments unlock the Victorian asylums and allow a more humane attitude to mental illness, they also led to the founding of modern neuroscience approaches to understanding the function and dysfunction of the most complex object in the universe- the human brain. This first wave of drug discovery and development took place in the absence of any meaningful knowledge of the underlying biological mechanisms, but rather occurred via serendipity coupled with careful observation. First generation compounds were consumed in vast amounts and the nascent pharmaceutical industry became immensely wealthy as the need for major and minor tranquillisers prevailed. Yet people were not 'cured' and the drugs themselves had sometimes life threatening side effects as well as the risk of addiction and dependence. It took many years to understand mechanisms of action to the point where drugs could be designed to target specific pathways and targets. Eventually, mechanisms of action were unveiled and there followed a period when effort was concentrated on the removal of undesirable side effects. As the key molecular targets became better understood, rivalry between companies to obtain a competitive advantage intensified and the focus switched to improving efficacy. As the low hanging fruit were rapidly picked, financial competition lead not to better drugs, but to mergers and acquisitions until it was realised that such approaches were more destructive than creative; and important skill sets were being discarded in the name of management efficiency.

As the scarcity of new compounds was realised, the genomic revolution swept in and promised a plethora of new molecular drug targets, with the hope that statistical association with a disease would provide sufficient justification for the allocation of the resources needed to bring compounds to market. It was hoped that psychiatric diseases would be monogenic; a single gene mutation which, once identified, would refocus the objective to finding a means of overcoming the deficiency or calming the over-activity. However, intense complexity has prevented unambiguous identification of genes and/or molecular targets for mental health disorders, as has happened to some extent in other fields, such as oncology or cystic fibrosis. Psychiatric disorders have been shown to be highly polygenetic with significant biological impact coming from genes, the environment and the unique life histories of the individuals. Furthermore, there is increasing evidence that many psychiatric disorders, for example depression and schizophrenia, are not single disorders but rather encompass a range of phenotypes that differ in causation and therefore will most likely require a different treatment approach.

The best example of this drug discovery model is the loss of function mutation of the SCN9A gene, which encodes the voltage gated sodium channel Nav1.7 and causes congenital insensitivity to pain in both humans and mice in which the gene has been experimentally deleted [1,2]. Accordingly, small molecule inhibitors of Nav1.7 were sought, especially since the null mutant mice otherwise appear healthy, suggesting such compounds, if selective, would be free of the cardiac side effects associated with broad spectrum sodium channel blockers [3]. Intense effort has indeed identified potent and selective compounds, but in animals, they provide only relatively weak analgesic activity e.g. ProTx-1l [4], and even neutralising monoclonal antibodies provides only partial and transient pain relief [5]. The reason for this discrepancy between theory and practice is a timely lesson to remember that life did not evolve to serve the interests of pharmaceutical companiesbiological mechanisms are always more complex than we like to think. Despite the inconvenience, our capacity to understand that complexity is impressively demonstrated by the work of scientists at University College London, who demonstrated the reason behind this weak analgesic effect, despite potent Nav1.7 inhibition, is due to profound alterations in gene expression as a result of the mutation, leading to an upregulation of endogenous opioids [6]. For that reason, Minett et al. used the opioid receptor antagonist naloxone in Nav1.7 mutant mice and substantially reversed the behavioural analgesia. Remarkably, Minett et al. had access to a human subject with SCN9A deletion in which there was a dramatic reversal of analgesia on infusion of naloxone. This work is a prime example of how we have moved a long way from the serendipitous discoveries of the past; the complex reasons behind the failures of today now provide a breeding ground for ideas and new knowledge to pave the way for future discoveries. 
An analysis of successes and failures within the global pharmaceutical industry by Morgan et al. highlighted the pitfalls we often fall into. On analysis of 44 programs from Pfizer, the majority failed to demonstrate efficacy; and a large number of cases $(43 \%)$ actually failed to adequately test their hypothesis. From these investigations, three key factors were found to clearly contribute to failure; i) lack of exposure at the site of action, ii) lack of target binding, and iii) lack of functional pharmacological activity [7].

Recent advances may enable us to tackle these problems, however highly specialised expertise and of course, adequate funding is required. Nevertheless, new preclinical technologies allow for valid, translational and relatively cheap ways to face the problem:

In the first instance, a key hurdle is that studies are not always backed up by pharmacokinetic evidence that the dose administered will provide appropriate tissue concentrations at the molecular target. Proton Emission Technology (PET) studies using selective radioligands may go some way to tackle this problem, by clearly showing that the compound enters the brain and occupies its receptor or target.

Next, it needs to be demonstrated that occupation of the target by the drug results in a pharmacodynamic effect. To show functional activity after target engagement requires a biomarker that indicates the system of interest has been pharmacologically activated. From a human neuropsychiatric perspective, functional neuroimaging such as fMRI BOLD would be the technology of choice, as it is able to demonstrate the brain structures associated with activation of the molecular target. Furthermore, fMRI also allows the subject to be monitored whist simultaneously performing a task relevant to the disease of interest, and thus potentially providing the beginnings of target validation.

Accomplishing this in small laboratory animals, however, is complicated by the necessity to minimise movement by using anaesthetic, which may subsequently confound results. Nonetheless, since the BOLD measure is dependent on changes in tissue oxygen concentration, this can be determined in small brain regions in freely moving behaving rodents using the technique of oxygen amperometry [8]. Here, a small carbon electrode is implanted into a region of interest and when a potential of -0.75 millivolts is applied, tissue oxygen is oxidised and the resultant electron flow is proportional to the concentration of oxygen. Using an appropriately designed tether, the animal can be placed in a suitable environment where stimuli can be applied and responses can be monitored and quantified. If the animal is in an operant box it can be trained to press a lever and obtain a food reward. This can be equated to a monetary incentive task which in the human BOLD paradigm leads to activation of the nucleus accumbens, a signal that is magnified when the value of the reward is increased [9]. Similarly in rodents, oxygen concentration is increased when the reward is increased from one food pellet to two [10]. Even more interesting, the slow $90.1-100 \mathrm{~Hz}$ inter regional oscillations that characterise the default mode resting state are also seen with oxygen amperometry, with nodes and edges very similar to the connectivity patterns seen in anaesthetised rats undergoing
BOLD measurements. Even more striking from a translational perspective are the similarities in drug induced changes to the strength of interregional coherence seen in the rat, compared to those seen in man. For example, it has been shown that ketamine enhances the connectivity between the anterior cingulate cortex and the hippocampus of man and rodents and the strengths wax and wane as the animal is signalled to pay attention to changes in reinforcement schedule [11]. Indeed, the unique effects of ketamine (via stimulation of AMPA receptors) are well worth investigating for its antidepressant activity, as predicted some time ago by its efficacy in the reviled mouse forced swim test $[12,13]$.

The more it becomes possible to make detailed investigations of brain circuitry involved in drug and behavioural responses, the greater the translational validity of clinical and preclinical proof of concept investigations will be.

Once it has been determined that a compound is present at the receptor, binds with sufficient affinity and has a biological effect, even then there are hurdles to face in the interpretation of the results. The quantification of rodent behaviour has long been thought a weak point of psychiatric drug discovery. However, despite the ongoing queries over the translational relevance of some behavioural tasks [14], recent evidence shows the results themselves do have excellent reproducibility, when experiments are carefully managed and replicated in different laboratories [15]. A simple administrative approach to ensure good reproducibility going forwards might be to ask authors of all journal submissions to present a signed statement that the key findings of the paper have been replicated independently by other investigators in the lab.

There are clearly some great obstacles in the way of developing new psychiatric medications, however new innovative ways of approaching the puzzle could (and currently are) pave the way to a brighter future of drug discovery.

One concept which has invigorated the field, and is providing an exciting new spin, is the finding that many diseases have an inflammatory component and could be tackled by antiinflammatory compounds. For example, a schizophrenialike state can be induced in subjects who have generated autoimmune antibodies to the NMDA receptor and NMDA receptor antagonists have been used for many years as acute pharmacological rodent models of schizophrenia [16,17]. NMDA receptor immunity, at the very least, lends a strong degree of validity to this animal model, even more so if the antibody could be prepared in sufficient amounts to be used in the animal model for a potentially more accurate recapitulation of the disease.

Another huge new technology is the conditioning of patient cells into neurons, and the subsequent creation of personalised cellular disease models [18]. This approach enables comparison of metabolic and functional differences between those affected with a psychiatric disorder and models of unaffected individuals. Differences could be determined and investigated for metabolic and functional errors, which could lead to novel and validated drug targets. Scientists at the University of Luxembourg have 
now successfully created three-dimensional brain-like cultures from skin samples. These cultures display different cell types which develop, connect, signal and exchange metabolic products, just like an active brain [19].

Finally, the potential for High Throughput Screening (HTS) as a drug discovery tool is becoming more apparent as a powerful route towards greater functional selectivity and clinical efficacy. The sorry tale of cortico-releasing factor (CRF) receptor antagonists is a prime example of how HTS may be necessary to make any headway in some cases. Multiple attempts with high quality CRF receptor antagonists have failed to show antidepressant efficacy [20-22], despite considerable evidence supporting the importance of the pituitary-adrenal axis in the pathophysiology of the disease [23]. Although there is much to support the potential of these compounds to be efficacious in the treatment of anxiety and depression, clinical trials of compounds having good drug-like characteristics have all failed without a hint of efficacy. A study by Zhang et al, reasoned that it is the CRF receptors in a specific area of the hypothalamus (Para ventricular nucleus) which are markedly involved in controlling CRF secretion [24]. Zhang et al. reasoned that global blockade of CRF receptors throughout the brain might obscure effects and they set about to genetically engineer the deletion of the CRF receptor specifically in the para ventricular neurons of the hypothalamus. The CRF receptor was depleted only from a group of cells expressing the transcription factor NIN1 , which is necessary for hypothalamic development $[25,26]$. This selective deletion gave a very robust anxiolytic phenotype, which may explain the lack of clinical efficacy after global CRF blockade. The question now is to identify a way of screening for antagonists possessing affinity only for the NIN-1 containing cells. It is possible that large volume screening of a mega sized sample collection could reveal such a compound.

Another great example of the promise of HTS is PZM21, an opioid agonist with major differences from all that has gone before- all the analgesic power of the opioids without respiratory depression. PZM21 was found by screening for novel opioid receptor agonists whilst looking for a compound devoid of action on respiratory pathways from a set of 3 million commercially available compounds [27]. Addiction potential has yet to be defined, but it shows the HTS approach can yield success. Likewise, the same logic could be applied to the metabotropic glutamate allosteric modulators which have very exciting in vivo behavioural profiles as potential anti-schizophrenic and wake promoting pro-cognitive molecules, but that cause cortical necrosis [28].

By screening out unwanted properties through concentrating on a very large sample of different molecules, we mimic the serendipitous approach of earlier times. However, this time, we base the screening on much stronger target validation by knowing beforehand what it is we do and do not want. If target validation has been done diligently, the chances of achieving positive effects in a human Proof of Concept experimental medicine study will be much higher and faster than ever before. Especially if all the lessons about optimising dose, providing evidence of target engagement, and of target activation/inhibition are heeded. With the availability of techniques like oxygen amperometry, a fully translational animal neuroimaging Proof of Concept study can be performed. Even analysis of local field potential recording, or unit activity, has now progressed to the point that drug induced engagement of circuitry can be confirmed in preclinical experimentation, saving both time and cost.

There is a general sense of gloom surrounding psychiatric drug discovery at present. Since those golden days of the 1950s, pharmaceutical companies and universities alike have strived to develop new drugs with improved therapeutic profiles and reduced side effects. Unfortunately, this process has not advanced as fast as it should, partly reflecting the complexity of brain disorders, the lack of meaningful 'biomarkers', an emphasis on developing drugs that predominantly duplicate the action of existing drugs and a reliance on dated and simplistic animal models. The scope and investment in psychiatric drug discovery research is highly sensitive to success, and of late there has been very little. Today, there is a deep rooted pessimism that without new focus and drive, the next generation of pharmaceuticals cannot be found; the trees have been stripped of their low hanging fruit. As a result, many companies have withdrawn or reduced the size of their psychiatric drug discovery teams. Despite this, we would argue that now is the wrong time to withdraw- the cost of brain disorders in terms of human suffering and economic burden to society is too high, and is rising.

The pharmaceutical industry will not inspire the next generation of investigators by walking away from difficult scientific problems. It is hoped that this short review will lessen the pessimism surrounding psychiatric drug discovery and contribute to attracting the pharmaceutical industry back into the field. Though still risky and expensive, the CEOs would do well to remember the huge financial and societal gain from those initial compounds, which were vastly inferior to what could become available in the future if the best new technologies are correctly exploited, and we have faith in those scientists who have dedicated their lives to this field.

\section{References}

1. Cox JJ, Reimann F, Nicholas AK, et al. An SCN9A Channelopathy Causes Congenital Inability to Experience Pain. Nature. 2006;444(7121):894-98.

2. Gingras J, Smith S, Johnson D, et al. Global Nav1.7 KO Mice Recapitulate Phenotype of Congenital Indifference to Pain Reported in Humans. J Pain. 2013;1:S44.

3. Walia KS, Khan EA, Dong HK, et al. Side Effects of Antiepileptics--a Review. Pain Pract. 2004;4(3):194-203.

4. Schmalhofer WA, Calhoun J, Burrows R, et al. ProTxII, a Selective Inhibitor of NaV1.7 Sodium Channels, Blocks Action Potential Propagation in Nociceptors. Mol Pharmacol. 2008;74(5):1476-84.

5. Jun-Ho L, CK Park, Chen G, et al. A Monoclonal Antibody That Targets a NaV1.7 Channel Voltage Sensor for Pain and Itch Relief. Cell. 2014;157(6):1393-404.

6. Minett MS, Pereira V, Sikandar S, et al. Endogenous Opioids Contribute to Insensitivity to Pain in Humans and 
Mice Lacking Sodium Channel Nav1.7. Nat Commun. $2015 ; 8967$.

7. Morgan P, Van Der Graaf PH, Arrowsmith J, et al. Can the Flow of Medicines Be Improved? Fundamental Pharmacokinetic and Pharmacological Principles toward Improving Phase II Survival. Drug Discov Today. 2012;17(9-10):419-24.

8. Lowry JP, Griffin K, McHugh SB, et al. Real-Time Electrochemical Monitoring of Brain Tissue Oxygen: A Surrogate for Functional Magnetic Resonance Imaging in Rodents. Neuroimage Clin. 2010;52(2):549-55.

9. Knutson B, Gibbs SEB. Linking Nucleus Accumbens Dopamine and Blood Oxygenation. Psychopharmacology. 2007;191(3):813-22.

10. Li J, Martin S, Tricklebank MD, et al. Task-Induced Modulation of Intrinsic Functional Connectivity Networks in the Behaving Rat. J Neurosci. 2015;35(2):658-65.

11. Gastambide F, Taylor AM, Tricklebank M, et al. Alterations in Spatial Memory and Anxiety in the MAM E17 Rat Model of Hippocampal Pathology in Schizophrenia. Psychopharmacology. 2015;232(21-22):4099-4112.

12. Hiroyuki K, Iijima MK, Chaki S. Involvement of AMPA Receptor in Both the Rapid and Sustained Antidepressantlike Effects of Ketamine in Animal Models of Depression. Behav Brain Res. 2007;224(1):107-11.

13. Maeng S, Zarate CA, Du J, et al. Cellular Mechanisms Underlying the Antidepressant Effects of Ketamine: Role of Alpha-Amino-3-Hydroxy-5-Methylisoxazole-4-Propionic Acid Receptors. Biol Psychiatry. 2008;63(4):349-52.

14. Homberg, Judith R. Measuring Behaviour in Rodents: Towards Translational Neuropsychiatric Research. Behav Brain Res. 2013;236:295-306.

15. Francois, Jennifer, Gastambide F, et al. Dissociation of mGlu2/3 Agonist Effects on Ketamine-Induced Regional and Event-Related Oxygen Signals. Psychopharmacology. 2015;232(21-22):4219-29.

16. Wandinger KP, Sandra S, Winfried S, et al. Anti-NMDAReceptor Encephalitis: A Severe, Multistage, Treatable Disorder Presenting with Psychosis. J Neuroimmunol. 2011;231(1-2):86-91.

17. Javitt DC. Negative Schizophrenic Symptomatology and the PCP (Phencyclidine) Model of Schizophrenia. Hillside J Clin Psychiatry. 1987;9(1):12-35.

18. Dolmetsch R, Geschwind DH. The Human Brain in a Dish: The Promise of iPSC-Derived Neurons. Cell. 2011;145(6):831-34.

19. Monzel AS, Smits LM, Hemmer K, et al. Derivation of Human Midbrain-Specific Organoids from Neuroepithelial Stem Cells. Stem Cell Reports. 2017; 318:1917-20.

20. Yavin S, Harriet de Wit. Lost in Translation: CRF1 Receptor Antagonists and Addiction Treatment. Neuropsychopharmacology. 2016;41(12):2795-97.

21. Spierling SR, Zorrilla EP. Don't Stress about CRF: Assessing the Translational Failures of CRF1antagonists. Psychopharmacology. 2017.

22. Zorrilla EP, George FK. Progress in CorticotropinReleasing Factor-1 Antagonist Development. Drug Discov Today. 2010;15(9-10):371-83.

23. Christine H, Newport DJ, Mletzko T, et al. The Link between Childhood Trauma and Depression: Insights from HPA Axis Studies in Humans. Psychoneuroendocrinology. 2014;33(6):693-710.

24. Zheng J, Babygirija R, Bülbül M, et al. Hypothalamic Oxytocin Mediates Adaptation Mechanism against Chronic Stress in Rats. Am J Physiol Gastrointest Liver Physiol. 2010;299(4):G946-53.

25. Duplan, MichaÃlle S, Boucher F, et al. Impact of Sim1 Gene Dosage on the Development of the Paraventricular and Supraoptic Nuclei of the Hypothalamus. Eur J Neurosci. 2009;30(12):2239-49.

26. Michaud JL, Boucher F, Melnyk A, et al. Sim1 Haploinsufficiency Causes Hyperphagia, Obesity and Reduction of the Paraventricular Nucleus of the Hypothalamus. Hum Mol Genet. 2001;14:1465-73.

27. Manglik A, Lin H, Aryal DK, et al. Structure-Based Discovery of Opioid Analgesics with Reduced Side Effects. Nature. 2016;537(7619):185-90.

28. Parmentier BS, Hutson PH, Menzel K, et al. Mechanism Based Neurotoxicity of mGlu5 Positive Allosteric Modulators--Development Challenges for a Promising Novel Antipsychotic Target. Neuropharmacology. 2014;82:161-73.

\section{*Correspondence to:}

Dr. Mark Tricklebank

Department of Neuroimaging

Institute of Psychiatry Psychology and Neuroscience

Kings College London

London

Tel: 02032283060

E-mail: mark.tricklebank@kcl.ac.uk 